\title{
TECNOLOGÍA DE PROCESOS: ALCANCES Y LIMITACIONES*
}

\author{
Jorge Flores Silva, Universidad Nacional Autónoma de Honduras, \\ Instituto de Investigaciones Económicas y Sociales (IIES UNAH), \\ Ciudad Universitaria edificio C2 primer piso. Tel./Fax: 504-22391849 \\ E-mail: jflores@iies-unah.org
}

\section{RESUMEN}

La tecnología de procesos y su implementación es básica en los distintos sectores de la economía.Su optimización para alcanzar las metas u objetivos de la empresa requiere que la tecnología no sólo sea la más novedosa, sino también que sea capaz de garantizar la sostenibilidad de la naturaleza en función de la vida.- En la medida que las tecnologías se desechen rápidamente, sus sustituciones pueden volverse anti-ecológicas, sino existe un proceso de sostenibilidad que lo acompañe.- Si el promedio de vida de la tecnología disminuye, también el promedio de vida del ser humano puede tender a disminuir debido a una posible disminución del promedio de vida de la naturaleza.-

E\&A

Si la tecnología de procesos solo toman en cuenta la máxima eficiencia para incrementar el rendimiento económico sin considerar los costos sociales y ambientales, sería una práctica irracional del uso de los recursos que desemboca en la autodestrucción.

La tecnología de procesos para evitar al máximo los males que acarrea la racionalidad formal que termina en irracionalidad debe estar fundada en la investigación.- Investigar para desarrollarse y no solo para crecer, desarrollo que debe estar fundado en la persona y en la naturaleza para la reproducción de la vida. En este caso, el conocimiento creado por la investigación no se agota, sino que queda acumulado y debe estimularse para la creación de ciencia y tecnología. Dicho conocimiento se va construyendo como parte de la práctica social.-

Palabras clave: Tecnología de procesos, Tecnología, Investigación

* W. Hughes (2003) "Para que su existencia sea permanente, todo proceso no solo debe producirse sino que, además debe reproducirse... No es posible recrear las condiciones de producción sin garantizar la reproducción de la vida humana” P.165 


\title{
PROCESS TECHNOLOGY: SCOPE AND LIMITATIONS
}

Jorge Flores Silva, Universidad Nacional Autónoma de Honduras, Instituto de Investigaciones Económicas y Sociales (IIES UNAH), Ciudad Universitaria edificio C2 primer piso. Tel. /Fax: 504-22391849

E-mail: jflores@iies-unah.org

\begin{abstract}
The implementation of process technology is essential for the different sectors of the economy. - The pursuit of goals of the company by the optimization of process technology requires that technology is the state of the art, but when technology is able to ensure sustainability. - To the extent that technology is quickly discarded, its replacement may become anti-ecological, if there is not a process of sustainability. - If the average life of technology decreases, the average of human life may tend to decrease due to a possible decrease on the average of life in nature.

If manufacturing processes only take into consideration the highest efficiency to enhance economic performance without considering social and environmental costs, it would be an irrational practice in the use of resources, leading to self-destruction.
\end{abstract}

In order to minimize the evils of formal rationality that ends into irrationality, process technology must be grounded in research. - This must be a research not only to growth for also to develop. This development must be based on the person and on nature for the reproduction of life. In this case, knowledge created by research does not run out, but it is accumulated for the creation of science and technology. Such knowledge is being built as part of the social practice.

Keywords: Investigation, Technology, Process Technology 


\section{INTRODUCCIÓN}

El incorporar tecnología implica hacer inversiones, asumir riesgos y amortizar recursos.- Son las grandes empresas las que generalmente modernizan sus unidades productivas, utilizando tecnología que en la mayoría de los casos desplaza a la mano de obra, problema que no resulta grave cuando se desarrolla el aparato productivo y se vitaliza la economía, pues los demás sectores como efecto del progreso y el desarrollo absorben la fuerza de trabajo desplazada.- El problema se da cuando por efecto de situaciones estructurales no se desarrolla la economía, y, la fuerza de trabajo desplazada como resultado del avance tecnológico no es absorbida por todos los sectores económicos.

La incorporación técnica como parte del incremento de las inversiones resulta ser de vital importancia para el crecimiento económico y la expansión de la capacidad productiva que incida sobre la base material de la sociedad y produzca un efecto sobre la economía en su conjunto.

Los procesos de innovación técnica tienen efectos primarios y secundarios asociados al incremento de la producción y la productividad, que inducen al crecimiento económico y al bienestar de la población, sin embargo, el tipo de tecnología y su carácter, presenta situaciones contradictorias

en los resultados que puede generar.- La sustitución de la fuerza física humana y energía animal, por la maquina, fue clave para considerar la época en que se dieron esos cambios, como la era del avance tecnológico, factor importante para el desarrollo económico.- De tal forma que, en la medida que se empleara mayor tecnología podría darse una relación positiva en la tasa de crecimiento, proporcionando las condiciones para que la población saliera de la pobreza y se revirtiera el estado de atraso, por mejores niveles de vida. La tecnología si se concibe como lo que es - un medio- puede contribuir al desarrollo integral, en cambio si la educación es tecnocrática, en donde la tecnología se ve como un fin difícilmente contribuirá a modificar pautas de comportamiento para vivir y saber convivir. Claro está que el crecimiento tecnológico expresado en recursos de aprendizaje contribuye a mejorar la enseñanza y asimilar conocimientos y a su vez la educación contribuye a este proceso para crear y asimilar la tecnología.

Según este planteamiento con solo incorporar tecnología se podría alcanzar un verdadero progreso económico y el bienestar de la población, sin embargo los resultados demuestran lo contrario.Los pequeños productores se han convertido en tan solo productores -consumidores, producir para 
consumir sin acceder a las relaciones mercantiles y cuando se accede se hace en función de la máxima ganancia porque media el interés particular.

Aunque la técnica deberá estar al servicio de todos los productores ha quedado demostrado según la experiencia histórica, que ésta con todos sus efectos depende de la estructura económica y diferenciación social de cada país.

Los propietarios de los medios de producción son los que realizan las inversiones, ellos tienen el dinero, de ahí que, quien posee el capital y los recursos naturales es el que tiene la facilidad para incorporar y utilizar tecnología, de esa forma los beneficios de ésta no se distribuyen equitativamente.

Estos son los conflictos y contradicciones que genera la tecnología, cuando funciona bajo la lógica de una racionalidad formal, que se centra en producir, teniendo como principios la eficiencia y la competitividad como valores supremos del mercado en función del interés particular, sin interesar el bien común y las necesidades generales de la sociedad. Revertir este proceso es el reto para poner la tecnología al servicio de la humanidad y al cuidado de la naturaleza.

\section{EL CONOCIMIENTO COMO BASE DE TODA TECNOLOGÍA DE PROCESOS}

El mundo globalizado busca la competitividad y la optimización, en donde la tecnología juega un papel importante para acelerar y mejorar los procesos que eleven el nivel de productividad como relación de eficiencia entre producto obtenido y factores de producción.

La capacidad de una empresa de mantenerse en las relaciones de mercado por sus condiciones de calidad y nivel de precios, es resultado en parte de la incorporación tecnológica a los procesos productivos y de gestión empresarial. Una empresa que esté mejor equipada puede lograr disminuir sus costos permitiéndole alcanzar razones financieras positivas y por lo tanto elevar su nivel de competitividad.

Hay una relación directa entre el nivel de productividad y competitividad: entre más alta es la productividad de una empresa es más competitiva. En todo esto la tecnología es fundamental no solo 
en relaciones técnicas de producción, sino sobre todo en la organización y gestión de los procesos para alcanzar los objetivos y metas. F. Hinkelammert (2003) "Celebramos la racionalidad y la eficiencia, sin embargo, estamos destruyendo la base de nuestra vida sin que este hecho nos haga reflexionar acerca de los conceptos de racionalidad correspondientes... Esta eficiencia ¿Es eficiente? Esta racionalidad económica ¿Es racional?... Las empresas logran una productividad del trabajo siempre mayor, si la medimos en relación con la fuerza de trabajo efectivamente empleada. Pero si relacionamos el producto producido con la fuerza de trabajo disponible, incluyendo en esta toda la población excluida, y si evaluamos los costos externos de la actividad empresarial, veremos que la productividad del trabajo se halla estancada, y es posible que incluso descendiendo. Así, pues, lo que parece progreso se esta transformando en un salto en el vacio”” P. 31. Al mismo tiempo Pablo Richard en teología de la solidaridad en el contexto actual de economía neoliberal de libre mercado señala "La eficiencia y la competitividad aparecen como valores absolutos, sin tener en cuenta la vida humana y cósmica como fin de toda actividad económica, tecnológica y cientifica” P. 224.

Se puede atribuir al mundo globalizado -en la que empresas y países se integren no solo por la vía del comercio sino también por el desarrollo e intercambio de tecnología- la vida del homo faber en la que se busca producir/consumir para satisfacer las exigencias del mercado.

Son indudables los beneficios de la tecnología de procesos o la tecnología para los procesos empresariales y/o de negocios. Observar, descubrir y aplicar ha sido la formula del avance científico-técnico que hoy se aprecia.

Sin embargo, estos avances no han surgido de la nada, siempre han necesitado las bases de viejos descubrimientos para gestar y/o dinamizar nuevos procesos.

Conocimiento-Tecnología - Conocimiento, es el camino circular en donde conocimiento y tecnología se retroalimentan, es decir el desarrollo de una implica el desarrollo de la otra.

Debe reconocerse que la base de todo proceso tecnológico es el conocimiento, el cual se va acumulando en el transcurso del tiempo a través del cual han surgido saltos cuantitativos y cualitativos de acuerdo 
a la base acumulada, por lo que todo conocimiento es relativo a los saberes anteriores.

El avance en la tecnología de procesos como resultado de descubrimientos, inventos o conocimiento nuevo causa cambios en las formas de trabajo y organización de las empresas, de ahí que la incorporación de la tecnología a los procesos forma parte de la fuerza productiva para disminuir costos, aumentar ganancias e insertarse con algún relativo éxito a las fuerzas del mercado.

Independientemente del reconocimiento o no del papel fundamental que juega la tecnología en el proceso productivo y de gestión de los negocios, no se avisora que el trabajo humano con su fuerza e ingenio pueda ser sustituida por la maquina. Es el ser humano quien diseña la maquina, la opera y la hace producir. El reconocer que el conocimiento se constituye como un factor de producción es reafirmar que el ser humano es insustituible, porque es el que organiza y ejecuta la actividad económica y hacia él se orienta para garantizar su existencia. El ser humano es el que crea el conocimiento y hacia él se dirige.

La tecnología solo es uno de los tantos medios que se ha ido desarrollando para perfeccionar los procesos e incrementar la productividad que induce al crecimiento económico.

E\&A

De manera que la tecnología y el conocimiento solo pueden darse como resultado del propio desarrollo humano. La revolución informática como salto innovador de las últimas décadas del siglo XX y principios del XXI, no es más que el reflejo del desarrollo de las fuerzas productivas en las que el trabajo del hombre con su capacidad creadora e innovadora es esencial.

\section{INVESTIGACIÓN Y TECNOLOGÍA RACIONALIZADORA}

El mundo moderno ha visto un avance en la actividad tecnológica, que ha facilitado las operaciones y ha generado ganancias por la vía de la reducción de costos, desde esa perspectiva ha sido un fenómeno que ha influido en cierta forma con el progreso de los pueblos. Es decir desde el punto de vista de la ganancia ha sido bueno porque ha generado riqueza material. Sin embargo desde el punto de vista del contenido ha significado retroceso porque se produce riqueza destruyendo las fuentes que generan riquezas es decir deteriorando el entorno, la biodiversidad.

La tecnología de procesos para evitar al máximo los males que acarrea la racionalidad formal que termina en irracionalidad debe estar fundada en la investigación. Investigar para desarrollarse y no solo para crecer, es decir investigación tecnológica para impulsar y mejorar sistemas de producción que implique eficiencia, competitividad a través del mejoramiento de los estándares de calidad, pero 
al mismo tiempo que permita evitar la destrucción de naturaleza que es de donde se extraen la materia prima, que el ser humano necesita para transformarlas.

Si los procesos de fabricación solo toman en cuenta la máxima eficiencia para incrementar el rendimiento económico sin considerar los costos sociales, se estaría ante una práctica irracional en el uso de los recursos que deshumaniza.

La investigación como elemento fundamental en la tecnología de procesos, no puede soslayar el hecho de que, para garantizar la existencia de materia prima para producir, no se puede destruir lo que es fundamental para la vida: la naturaleza. Esta responsabilidad debe llevar al empleo de una tecnología, que vista como lo que es, un medio, sea apropiada para el uso racional de los recursos que implique el mejoramiento de las condiciones de vida de las personas y no solo de la máxima ganancia.

En la medida que se producen bienes con una vida útil corta, como resultado del estimulo de los deseo que invita a producir y consumir, más rápido se destruye la naturaleza. Esto es una reflexión de profundo contenido, cuando se comprende que la naturaleza tiene límite y puede ser destruida, con consecuencias nefastas para la humanidad. Ante esta amenaza global vale la pena hacer referencia a la ecoeficiencia en los procesos productivos concepto que utilizan los especialistas en sus reflexiones sobre como producir más con menos, al respecto Valderrama, Liduvina y Díaz Martín, Diego señalan “la visión central de la ecoeficiencia se puede resumir en 'producir más con menos'. Utilizar menos recursos naturales y menos energía en el proceso productivo, reducir los desechos, atenuar la contaminación, es definitivamente positivo para el ambiente, y a la vez, resulta beneficioso para la empresa porque sus costos de producción y operación disminuyen.

Como meta final, la ecoeficiencia busca la elaboración de bienes y la prestación de servicios a precios competitivos que satisfagan las necesidades humanas y eleven la calidad de vida de la población. Al mismo tiempo, debe promover la reducción progresiva del impacto ambiental negativo de los productos, y procurar que su confinamiento dentro de la capacidad de carga de la tierra."

La tecnología de procesos y su implementación es básica en los distintos sectores de la economía. Su optimización para alcanzar las metas u objetivos de la empresa se dará no cuando la tecnología caiga en la obsolescencia, sino cuando sea capaz de garantizar la sostenibilidad de la naturaleza en función de la vida. En la medida que los conocimientos y prácticas tecnológicas se desechen rápidamente, se vuelven antiecológicos. Si el promedio de vida de la tecnología disminuye, también el promedio de vida del ser humano tenderá a disminuir porque disminuye el promedio de vida de la naturaleza. 
De igual forma se puede decir del conocimiento el cual se va construyendo como parte de la práctica social. En este caso el conocimiento no se agota sino que queda acumulado y debe estimularse para la creación de ciencia y tecnología.

Desechar el conocimiento es perder identidad corriéndose el riesgo de crear contra valores. La información única, la imagen única y el modelo único van desechando el conocimiento precedente de los mayores. En la medida que se desarrolla la industria, la agricultura y el comercio se exige más rendimiento de los factores de producción, esto solo es posible con el conocimiento de los trabajadores y de su capacidad técnica para operar las maquinas. Así como avanza el desarrollo del conocimiento de manera general, las exigencias de las distintas áreas de la producción y los servicios también demanda de competencias especificas.

Los procesos tecnológicos se orientan a elevar la productividad para ser más competitivos esto indudablemente demanda de mayores conocimientos para ejecutivos y trabajadores, es aquí donde el sistema educativo juega un papel importante como generador de procesos humanos para el desarrollo de la sociedad a partir del desarrollo de las potencialidades humanas.

E\&A

El mundo actual que globaliza los hábitos de consumo y de las formas de hacer negocio como resultado del avance en las telecomunicaciones, hace que todo se transforme rápidamente cambiando al mismo tiempo los estilos de vida y las formas de adquirir el conocimiento.

El ambiente ha sido invadido por el relativismo lo que ayer era verdad hoy ya no lo es. No solo se tienen distintas percepciones de la realidad como es natural, sino que la verdad se distorsiona y se acomoda.

Se trabaja ya no para producir riqueza real sino para especular. Cuando más desempleo agobia a la humanidad principalmente a la de los países pobres, se afirma que con los nuevos procesos tecnológicos se trabajara más y se descansará menos. Esto resulta ser hasta cierto punto una contradicción.

Todo gira en torno a una visión técnica, cambiando no solo las modalidades y recursos de aprendizaje, sino hasta los contenidos y de todo aquello que se necesita aprender para privilegiar la cultura del “éxito". Lo de ayer ya no sirve hoy, menospreciando con ello los procesos que dieron vida a los métodos y formas de trabajo de hoy. Los conocimientos de la sociedad en su conjunto son la suma de los conocimientos de cada individuo, el desarrollo de los procesos tecnológicos se incrementan como resultado del conocimiento individual de cada uno de los que intervienen en el proceso. Por tanto el conocimiento que encierra cada sociedad como cada proceso, crece a un ritmo más acelerado 
que el de los individuos vistos éstos de manera particular en virtud que los conocimientos, las competencias, habilidades y destrezas de la sociedad y los procesos es la totalidad de los sujetos vistos individualmente.

\section{LA TECNOLOGÍA DE PROCESOS Y EL UTILITARISMO}

La cultura del cambio que se pregona esta asociada a la cultura del éxito y la prosperidad que excluye y margina. Seguir un pensamiento convencional desde esta perspectiva no solo implica perder oportunidades de insertarse con éxito a la economía global. La orden del día que estimulan los nuevos procesos es la del utilitarismo del aparato productivo para poder sobrevivir en el mercado.

Se exige cambio de mentalidad ajustado al cambio que vive el mundo actual, el cual es resultado de procesos que implican no solo choque de generaciones, sino también choque de civilizaciones.

Estas aspiraciones del mercado total se hacen posibles a través de los curriculum educativos de los centros de enseñanza, especialmente las universidades y los programas de educación no formal de las empresas que orienta el mundo al "mundo del negocio". En todo esto la tecnología juega un papel importante. Tecnología vista como factor que hace posible el incremento de la productividad como resultado de la optimización en el uso de los recursos que siempre resultan ser escasos.

Invertir en un negocio o en la actividad productiva es para obtener alguna ventaja económica o social. Desde la óptica particular se espera un rendimiento económico, desde la perspectiva del sector público se espera un rendimiento social. Las empresas como sujeto económico y los empresarios como sujeto social buscan optimizar el uso de los recursos para lo cual incorporan progreso técnico.

El empleo de la tecnología de manera apropiada no solo es introducir la maquina, sino también implica impulsar nuevas formas de trabajo eficaces y eficientes. Los procesos tecnológicos implican mejora continua, generando un sistema de gestión y organización para alcanzar objetivos y metas como parte de la eficacia en el desempeño. Al mismo tiempo los procesos tecnológicos con la ayuda de la organización y gestión pueden lograr una relación positiva entre la producción obtenida y los recursos utilizados como parte de la eficiencia.

Trabajar con eficiencia y eficacia utilizando nuevos procesos es estar a tono con los nuevos tiempos. Implica una mentalidad abierta al cambio para asimilar las nuevas tecnologías y los nuevos procesos 
que incorporen valor, ventaja económica y ventaja competitiva a las unidades de producción y al producto generado. Desde luego que estos propósitos se logran no con una planificación puramente formal o con estructuras que no responden a las aspiraciones de los que inicialmente realizaron una inversión. Es necesaria una efectiva coordinación intra e inter institucional que oriente de manera coherente la planificación estratégica.

La planificación estratégica ha de tener repercusión en el tiempo, es a corto, mediano y largo plazo que se deben diseñar los planes de negocio, planes de inversión, análisis de costos, adquisición o impulso de tecnología informática, planes ambientales y de ecoproduccion.

Las empresas y las instituciones han de estar continuamente innovándose, la tecnología de las comunicaciones y la tecnología de procesos debe orientar sus esfuerzos a la creación y aplicación de nuevas técnicas y conocimientos que den respuesta no solo a los problemas inmediatos de la empresa y/o de los clientes, sino también a la problemática que atañe a la sociedad. Entre estos problemas están el manejo de las cuencas hidrográficas, el manejo de los desechos sólidos, la contaminación ambiental, mala práctica en el procesamiento de la materia prima, rendimientos bajos de las empresas,

E\&A insolvencia y baja productividad. En tal sentido impulsar la investigación ha de ser una práctica no solo de las universidades, sino también de la diversas unidades económicas que implementen procesos para la eficiencia y la eficacia.

\section{LA EDUCACIÓN Y EL LIDERAZGO DE LAS EMPRESAS}

La investigación como parte de los procesos técnicos contribuye al desarrollo de la inteligencia, pero este desarrollo es parte también de la experiencia que se ejecuta con la actividad práctica. Los procesos técnicos resultado del ingenio humano, también de manera circular han de contribuir al desarrollo de ese ingenio. En la medida que se desarrolla el ingenio resultado de las necesidades y de los procesos que van surgiendo con el crecimiento de la población, el agotamiento de los recursos y la división del trabajo, se va apreciando el potencial humano. Es el trabajo mismo del individuo que lo hace adquirir destrezas. Algunas habilidades no se enseñan en los centros educativos sino que son resultado de procesos tecnológicos y de gestión que marcan la experiencia laboral en el centro de trabajo.

El desarrollo de las fuerzas productivas y la compleja división del trabajo en la que van predominando las redes de producción, la educación juega un papel importante en la tecnología de procesos y en la 
planificación de esos procesos.

Desde la perspectiva macroeconómica y no solo microeconómica es necesario la tecnología de procesos, para una intervención racionalizadora del Estado en la conducción económica. También es importante la planificación del proceso educativo para racionalizar procesos y recursos.

La tecnología de procesos en la educación es básica en la planificación de la educación para la planificación de la economía, esto implica entonces una tecnología de información que lleve a potenciar fortalezas y oportunidades, superando las debilidades y eliminando las amenazas.

La investigación como parte de la tecnología de procesos y la tecnología de procesos como resultado de la investigación, debe permitir conocer la realidad observable y el mundo complejo en que se implantan las empresas y se desenvuelve el individuo. Esto demanda del dominio de una disciplina y cultura científica aunque sea de manera general para convertirse en factor clave del desarrollo.

Este dominio solo será posible si al mismo tiempo se brinda una información y formación adecuada a los responsables de la organización, gestión y operación de las empresas. De tal manera, no se puede descuidar la atención del recurso humano que orienta los procesos. En este sentido la tecnología de procesos debe formar parte de un proceso deliberado, para influir racionalmente en la optimización de recursos y obtener así rendimientos que reporten ventaja económica y social.

Deloitte" "En la era de las tecnologías de información... ...las empresas" buscan "Cómo optimizar la utilización de los recursos tecnológicos para generar valor y ventajas competitivas en el desarrollo del negocio, diseñando procesos mas eficientes y eficaces, apoyando la gestión interna y la forma de relacionarse con la cadena de valor de la empresa... ...en el proceso de cambio que esto significa”, se ofrecen "un conjunto integral de servicios:

- Planificación estratégica y reestructuración organizacional

- Diseño y elaboración de estrategias y planes de tecnología

- Modelamiento y rediseño de procesos de negocios

- Control de gestión e inteligencia de negocios

- Apoyo en la gestión de costos

- Diseño y gestión de proyectos informáticos

- Conducción de procesos de adquisición de infraestructura y servicios informáticos

- Selección de herramientas tecnológicas

- Implementación de paquetes de software"

1 www.deloitte.com/dtt/section_node 
Los anteriores elementos constituidos como servicios vienen a ser factores básicos para lograr competitividad e insertarse con éxito en las relaciones de mercado. El liderazgo de las empresas se lo da el grado de empuje que manifieste no solo en la prestación de servicios, sino también del tipo de producto que ofrezca para satisfacer al cliente. La calidad que esta referida a las exigencias del público no puede pasar por alto los procesos de transformación asociado al manejo ambiental. Esto es valido para empresas de cualquier sector que desarrolle transformación agrícola, industrial o agroindustrial. Buenas practicas, calidad de productos y servicios y uso apropiado de los recursos naturales marcaran el liderazgo de la empresa. No se puede ser líder en el mundo de los negocios cuando la empresa no considera lo que los economistas llaman las externalidades. Los bienes y servicios que se ofrecen satisfacen una necesidad inmediata pero han de tener una repercusión social.

Uno de los ejemplos más claros sobre los servicios y su efecto social lo constituye la educación, a menudo el analista económico se pregunta ¿Cuál es la inversión más importante? Aquella inversión de capital que puede generar una ventaja económica inmediata como resultado de la combinación de los factores de producción o aquella que implica aumentar el saber científico en la persona para perfeccionar sus habilidades técnicas y adquirir las competencias necesarias para desempeñarse en el trabajo. Hacer una inversión demanda de un estudio previo, al menos es lo habitual en toda empresa y/o institución que trabaja organizadamente y que planifica para evaluar objetivos y resultados.

Hay variados criterios para hacer la inversión, todo va depender de la visión que se tenga de los problemas del desarrollo, del grado de crecimiento que se estime para la empresa, de las expectativas y del tiempo que se espera recuperar la inversión.

El empresario particular desea recuperar rápidamente el capital invertido, su visión es micro y esta en función de su ganancia. La educación es una empresa para toda la vida en la que la persona aprende desde que nace hasta que muere, que habilita no solo para un trabajo sino para vivir y comunicar la vida, resulta en un renglón estratégico que preferentemente debe, sobre ella, tenerse una visión macro. La visión micro interesa la rentabilidad económica, la visión macro interesa la rentabilidad social. Desde una perspectiva empresarial en la que solo se piense en la ganancia inmediata y en la recuperación de la inversión en el menor tiempo posible, no se toman en cuenta las externalidades. Educarse prepara y sirve para que la persona se realice en el plano individual y en el ámbito social. De manera que los beneficios de la educación no se circunscriben a la persona que recibe de forma directa los conocimientos, también la familia, los compañeros de trabajo y la sociedad en que se desenvuelve 
el individuo educado en su interrelación social obtiene frutos positivos que se traducen en rentabilidad social. Estos beneficios sociales casi no los toma en cuenta en sus consideraciones económicas el que invierte en educación en el plano privado, porque no hay una rentabilidad inmediata de manera tangible en el corto plazo. Transmitir el conocimiento y valores a través de la tradición o en el plano de las relaciones laborales pasa desapercibida, sin embargo es ahí donde esta el efecto social externo a la persona que directamente recibe la educación.

Un gerente bien preparado tanto humanista como técnicamente, tendrá resultados positivos sobre los demás trabajadores y empleados, que se traducirán en incremento de la productividad de la empresa, por efecto del conocimiento en las relaciones laborales y demás factores relacionados con la organización y gestión empresarial.

\section{MULTIDIMENSIONALIDAD DE LA TECNOLOGÍA DE PROCESOS}

El carácter multidimensional de la tecnología de procesos que no se circunscribe a la organización y gestión para facilitar las operaciones, mejorar los sistemas de entrega y optimizar recursos en el área administrativa también aporta mecanismos de innovación para la transformación industrial y agrícola a través de procesos técnicos que no solo son de relaciones entre personas como organizadores y ejecutores de la producción sino también de transformación de materia prima y recursos naturales. Sin embargo W. Hughes (2003) “... la forma en que se pretende impulsar el desarrollo... ... donde las leyes del mercado adquieren la supremacía de la regulación del funcionamiento del sistema económico, se mantiene el criterio de rentabilidad como el núcleo de las decisiones económicas, ... ... tal esquema de "desarrollo" es incompatible con una visión del desarrollo que anteponga la vida de la gente al crecimiento económico (y a la producción de ganancia), ... ” P. 164

En el campo agrícola usando una tecnología conservadora de los recursos naturales cuyo bien ha de ser el uso racional del principal medio de producción -la tierra-.

El uso extensivo de la tierra limita la incorporación de progreso técnico, en tal sentido la rentabilidad de la tierra y de los cultivos agrícolas puede lograrse usando más intensivamente los factores de producción, incorporando estímulos productivos basados en la utilización intensiva de insumos tecnológicos como la semilla mejorada, fertilizantes plaguicidas y otras que incrementan la rentabilidad agrícola sin desplazar mano de obra. Una tecnología desplazadora de fuerza de trabajo como la que se da con la introducción de maquinaria y equipo pesado propio de las grandes extensiones, resulta menos viable que la tecnología conservadora de explotación intensiva que emplea tecnología 
apropiada mas común en la pequeña explotación.

El manejo de tecnologías apropiadas y la introducción de nuevas practicas de cultivo debe ser el resultado de una política tecnológica basada en la responsabilidad ética ante la naturaleza, que implique introducir cultivos que sean compatibles con el bosque pero sobre todo el empleo de una tecnología ahorradora de recursos y generadora de trabajo para el excedente de mano de obra propio de los países subdesarrollados.

Aprovechar las ventajas competitivas, sobre todo la riqueza natural y el potencial productivo del recurso humano, sobre la base de tecnología de procesos innovadores es tarea urgente para las empresas agrícolas y los países en los que la agricultura contribuye de manera significativa a la economía en su conjunto. H. Forján² “, ...las tecnologías de procesos presenta un fuerte componente de información, conocimiento y eficiencia incorporada, con una aplicaron casi personalizada por parte del productor. Más que un costo económico tienen un costo intelectual y un costo en tiempo, y su adopción es más dificultosa. Muchas empresas agropecuarias están llevando adelante este tipo de tecnologías que no son necesariamente dependientes de la incorporación de insumos.

Las tecnologías de procesos son aquellas que requieren de un trabajo de programación previo, una presencia, un aprendizaje y un control o seguimiento casi permanentes. Entran en esta categoría todas las tecnologías de manejo (de cultivos, de suelos, de plagas, de pasturas, de rodeos, etc.) y el gerenciamiento de actividades mas complejas como promover asociaciones (para producir, comercializar, etc.) a fin de mejorar la competitividad frente al mercado aceptando las nuevas tecnologías pero midiendo el posible efecto sobre todo el sistema de producción"

De lo anterior se puede aseverra que la tecnología de procesos es una practica que se aplica en todas los sectores de la economía. Puede generar beneficios o perjuicios según se le conciba como un medio o como un fin. Concebida como lo que es, un medio del proceso económico, aumenta la eficacia de las operaciones y la productividad y al mismo tiempo garantiza la sostenibilidad de los recursos para garantizar la vida de las personas que son el sujeto del desarrollo. Buscar valor para las empresas sin menospreciar el valor de las personas, ahorrando tiempo, reduciendo costos, administrando información sin actuar arbitrariamente sobre la naturaleza es el reto al que se ven avocados las unidades de producción. En esta tarea es fundamental la investigación para desarrollar procesos que incorporen tecnologías ahorradoras no para producir riqueza destruyendo las fuentes generadoras de riqueza, sino para garantizar la vida de las personas y el planeta tierra como la casa de todos. Pasar 
de las ideas a las realizaciones prácticas es tarea urgente. La investigación no debe circunscribirse a aquella que realizan los institutos especializados. El mundo actual y el modo de funcionar de la economía demandan de altos niveles de formación profesional para participar en el complejo campo de las relaciones personales, sectoriales e interfactoriales.

Asimilar los avances de la tecnología y procesos de información rápida exige de estos altos niveles de preparación, que permitan adaptarse a la era de la complejidad y la incertidumbre para tomar las mejores decisiones. Aunque hay instituciones privadas y organizaciones no gubernamentales que realizan investigación, son las universidades el espacio mas adecuado para la teoría y practica científica, que da mayor sentido a su trabajo como entidad de educación superior. Para que haya creación de ciencia se necesita del científico, el profesor docente sobre todo el de educación superior debe encaminarse por este perfil porque es en las universidades donde se instruyen los que han de preparar a los formadores, los científicos, los administradores y los que han de dirigir los procesos y los destinos de la sociedad.

Todavía no se alcanza a comprender que las universidades son instituciones de desarrollo donde se crea ciencia y tecnología. Solo desarrollando la investigación se puede formar un profesional intelectualmente maduro para impulsar procesos técnicos que lleven a las empresas a gestionar mejor los recursos, alcanzar la productividad y ser más competitivos en el mundo de los negocios.

\section{DESARROLLO DE LAS POTENCIALIDADES HUMANAS Y LA TEC- NOLOGÍA DE PROCESOS}

La innovación tecnológica y el avance científico demanda del desarrollo de competencias para abrirse campo ante las exigencias de la economía global.

Educación y desarrollo son dos caras de la misma moneda, la educación es elemento clave para el desarrollo y el desarrollo como elemento valorativo que incorpora los aspectos cualitativos que brinda la educación, ésta como factor que humaniza cuando se orienta no solo a adquirir ciertas habilidades y destrezas, como si se tratase solo de formar "técnicos" olvidando la responsabilidad de formar "personas". Continuar con modelos educativos tecnocráticos es ver a la persona únicamente como una unidad de producción o de consumo.

La racionalidad científico técnica de los nuevos procesos están llevando al ser humano a producir para consumir. La educación debe conducir a eliminar esas prácticas y a evitar las distorsiones en los procesos económicos si no se quiere caer en la deshumanización que afianza el subdesarrollo. 
Las teorías administrativas y de la producción que privilegian la competitividad, la eficiencia y la productividad en función de la máxima ganancia que sobrepone el capital ya no como medio sino como fin, deben ser objeto de estudio en el marco de una política educativa que tenga como objetivo elevar al ser humano en su dignidad como persona. El ser humano sea un obrero o un ejecutivo, un analfabeta o un ilustrado es ante todo persona. Persona con inteligencia, con voluntad y con libertad que a través del trabajo busca realizarse y participar en el proceso de desarrollo.

Educar para pensar debe ser un objetivo en todo proceso de enseñar y aprender. La motivación intrínseca del ser humano de saber, es lo que lleva a participar en el acto educativo. Esta tarea se encamina a comprender, a conocer, asimilar y no solo hacer. A menudo se cuestiona la educación acusándola de ser muy teórica, se afirma que de acuerdo a los nuevos contextos se debe ser más práctico adaptándola a los procesos productivos, a la demanda empresarial, en otras palabras al utilitarismo del mercado.

Si la educación es para saber pensar, cambiar comportamientos, realizar y humanizar ésta no solo se debe concebir desde la perspectivas procedimental, que en vez de crear las condiciones para la promoción humana solo instrumentaliza al individuo.

Los enfoques utilitaristas que generalmente observan los procesos técnicos y hacia los cuales se orienta la educación, se comportan envolventes para todo el sistema de enseñanza-aprendizaje, por eso la educación que se brinda invita a hacer cosas, se prepara al individuo para que vaya a hacer cosas que luego ha de consumir y como la educación alienta ese comportamiento en un modelo excluyente que desprecia al que "no tiene" y aprecia al "que tiene cosas" generando un conflicto de identidad. El mercado no lo es todo, los valores supremos del mercado que impone la economía global, deben verse como lo que son, medios para realizar al hombre que es el fin de la actividad económica. Urge formular una política educativa general con principios filosóficos y doctrinarios en correspondencia con un tipo de ciudadano que sepa responder a los desafíos del mundo actual. Gómez Buendía " $L a$ alta inteligencia conlleva una ética de aceptación de la verdad, de responsabilidad, de compromiso con el bien público por encima de los interese particulares o sectoriales, de respeto invariable por los valores universales encarnados en los derechos humanos y en la práctica de la justicia. Por eso es necesario desarrollar el pensamiento critico y estimular la actividad cientifica desde la primera escuela y a lo largo de toda la vida educativa" P.xxxvi.

La universidad cuyo papel no se circunscribe a formar profesionales, sino también a buscar la verdad 
científica, a través también del proceso de desarrollo del pensamiento científico y filosófico, debe enseñar a vivir en la verdad, encontrarse con la verdad, difundir la verdad y comprometerse con la verdad. Esto desde luego implica compromiso institucional que apoye una política de investigación como función básica y permanente de la universidad, para ello deben volcarse los esfuerzos académicos y financieros pertinentes que favorezcan el desarrollo de la ciencia y la innovación tecnológica de alto rigor. Núñez Jover, Jorge " "La imagen de la ciencia como una actividad de individuos aislados que buscan afanosamente la verdad sin otros intereses que los cognitivos, a veces transmitida por los libros de texto, no coincide para nada con la realidad social de la ciencia contemporánea. En gran medida el desarrollo científico y tecnológico de este siglo ha sido impulsado por intereses vinculados al afán de hegemonía mundial de las grandes potencias y a las exigencias del desarrollo industrial y las pautas de consumo que se producen y se difunden desde las sociedades que han marcado la avanzada en los procesos de modernización”, en este sentido, no todo lo que es científicamente posible es éticamente aceptable, el primer criterio ético de todo proceso debe ser guardar la racionalidad en función del ser humano. La crítica que se plantea en torno a la incorporación tecnológica reside no en la incapacidad de producir tecnología, sino en la incapacidad de llevar el progreso y aprovechar el desarrollo tecnológico para evitar el atraso. Es decir que con la llamada "era del conocimiento" con capacidad de producir tecnología, no se aplica ésta de manera apropiada, guardando la racionalidad para conservar la naturaleza y evitar el deterioro ambiental que minimice los efectos nocivos sobre la vida.

Desde esa perspectiva la economía y los actores involucrados en la tecnología de procesos y/o los procesos técnicos propios del proceso económico deben abordar los fenómenos tanto a nivel micro como macro, con amplio criterio científico, analizando el modo de funcionar del modelo económico, sus relaciones y los valores supremos que expresa, porque como apunta el profesor F. Hinkelammert (2003) "la teoría económica y social se torna cínica" en una "economía......en la cual se trata de conseguir ventajas competitivas ... .... y toda actividad social es evaluada de acuerdo con su aporte a esta competitividad" p.20.

La tecnología de procesos como parte integradora de un proceso educativo que humaniza y socializa debe ser el objeto propio de hacer ciencia. La verdad siempre será una, las percepciones de la realidad son las diferentes y en la medida que estas.

3 Organización de Estados Iberoamericanos, para la educación, la ciencia y la cultura. 


\section{REFERENCIAS}

1. Deloitte. -http://www.deloitte.com/dH/Section_node

2. Departamento de Tecnología de Procesos y Sistemas. UCA

3. Espino Cedeño, Maximino. -Para pensar y hacer la Educación. -Panamá, 1996

4. Forjan, Horacio. -http://www.inta.gov.ar/barrow/info/documentos/agricultura/rotaciones/tecno procesos.htm

5. Getronics. -http://www.uamcav.vat.edu.mx/notic/2005/03/docs/éxito_Empresarial.Pdf

6. Gómez Buendía, Hernando (Director). -Educación la agenda del siglo XXI: Hacia un desarrollo humano. -Programa de Naciones Unidas para el desarrollo. -PNUD. TM Editores. 1998

7. Hinkelammert, Franz. -El asalto al poder mundial y la violencia sagrada del imperio. - Colección Economía. -Teología. -San José, Costa Rica. 2003.

8. Hinkelammert, Franz y Mora, Henry M.- Coordinación social del trabajo, mercado y reproducción de la vida humana.- Colección Economía-Tecnología.- Editorial Departamento Ecuménico de Investigación (DEI), Costa Rica, 2001.

9. Hinkelammert, Franz J. El sujeto y la ley: el retorno del sujeto reprimido EUNA. Editorial Universitaria Nacional Heredia, Campus Omar Dengo. Costa Rica 2003.

10. Hinkelammert, Franz (compilador). El huracán de la globalización. Editorial Departamento Ecuménico de Investigaciones (DEI), de la edición en español, San José, Costa Rica, 1999

11. If People, innovación para la gente. -http://www.empresasustentable.com/services/tech/ bizprocess/bizbenefits

12. Labarca, Guillermo (compilador). -Economía Política de la Educación. -Editorial Nueva Imagen. -México. 1980

13. Núñez Jover, Jorge. -La ciencia y la tecnología como procesos sociales. -Lo que la educaron científica no debería olvidar. -Organización de Estados Iberoamericanos, para la educación, la ciencia y la cultura. - http://www.oei.es/salactsi/nunez05.htm

14. Principios de economía micro: Economía de la educación. -s.n.p.i

15. Tecnología de Proceso. -http://www.reiva/es/paginas/reiva/tecne.htm

16. Vitales. Valderrama, Liduvina y Díaz Martín, Diego. -http://www.vitalis.net/ecoeficiencia.htm 